\title{
Development and validation of a liquid chromatography-tandem mass spectrometry assay for the simultaneous quantification of buprenorphine, norbuprenorphine, and metabolites in human urine
}

\author{
Sherri L. Kacinko, Marta Concheiro-Guisan, Diaa M. Shakleya, and Marilyn A. Huestis \\ Chemistry and Drug Metabolism, Intramural Research Program, National Institute on Drug Abuse, \\ National Institutes of Health, Suite 200, Room 05A-721, 251 Bayview Blvd., Baltimore, MD 21224, \\ USA
}

\begin{abstract}
A liquid chromatography-tandem mass spec-trometry method for the simultaneous quantification of buprenorphine (BUP), norbuprenorphine (NBUP), buprenorphine glucuronide (BUP-Gluc), and norbuprenorphine glucuronide (NBUP-Gluc) in human urine was developed and fully validated. Extensive endogenous and exogenous interferences were evaluated and limits of quantification were identified empirically. Analytical ranges were $5-1,000 \mathrm{ng} / \mathrm{mL}$ for BUP and BUP-Gluc and 25-1,000 $\mathrm{ng} / \mathrm{mL}$ for NBUP and NBUP-Gluc. Intra-assay and interassay imprecision were less than $17 \%$ and recovery was $93-116 \%$. Analytes were stable at room temperature, at $4{ }^{\circ} \mathrm{C}$, and for three freezethaw cycles. This accurate and precise assay has sufficient sensitivity and specificity for urine analysis of specimens collected from individuals treated with BUP for opioid dependence.
\end{abstract}

\section{Keywords}

Buprenorphine; Norbuprenorphine; Buprenorphine glucuronide; Norbuprenorphine glucuronide; Urine; Liquid chromatography-tandem mass spectrometry

\section{Introduction}

In 2002, buprenorphine (BUP) became available in the USA for office-based treatment of opioid dependence under the Drug Treatment Act of 2000. This law allows physicians to write prescriptions for schedule III, IV, and V narcotic medications specifically approved by the Food and Drug Administration for narcotic addiction treatment. As the popularity of BUP increases for opioid dependence treatment and expands to high-risk populations such as pregnant women, there is an increased need for accurate and specific methods to monitor treatment compliance and to detect and quantify illicit BUP use [1-3].

Immunoassays to screen human urine [4-9] and gas chromatography (GC) systems coupled to a variety of detectors have qualitatively identified and quantified BUP, norbuprenorphine (NBUP), and glucuronides in urine [4,10-14]. Glucuronides are not easily detected by GC, making hydrolysis necessary to quantify total BUP and metabolites in urine. GC coupled with mass spectrometry (MS) also requires a derivatization step that may be difficult to fully complete for NBUP [15]. 
Liquid chromatography (LC) with tandem mass spec-trometry (MSMS) detection offers several advantages for the analysis of BUP and metabolites. Glucuronide concentrations may be directly measured by LCMSMS, eliminating the need for hydrolysis, and derivatization is not required. LCMSMS was applied to analysis of urine for BUP and NBUP [16-18], for conjugated metabolites [19], and for free and glucuronidated metabolites simultaneously [20, 21], but a complete and thorough validation addressing sensitivity, linearity, carryover, imprecision, recovery, extraction efficiency, matrix effect, process efficiency, matrix interference, dilutional integrity, selectivity, and stability was not published previously.

\section{Experimental}

\section{Chemicals and reagents}

BUP, NBUP, buprenorphine glucuronide (BUP-Gluc), norbuprenorphine glucuronide (NBUPGluc), $d_{3}$-NBUP, and $d_{4}$-BUP standards and internal standards were obtained from Cerilliant (Austin, TX, USA). Immunoassay quality control (QC) BUP was from Lipomed (Cambridge, MA, USA). Stock BUP-Gluc and NBUP-Gluc QC solutions were purchased from ElSohly Laboratories (Oxford, MS, USA). A separate NBUP manufacturer was no longer available; therefore, QC samples were prepared from a different lot of Cerilliant stock solution, when possible, or from a different vial, with preparation on different days than for calibrators. Reagent grade formic and perchloric acid were from Sigma Chemicals (St. Louis, MO, USA) and Acros Organics (Morris Plains, NJ, USA), respectively. All solvents were highperformance LC grade.

\section{Instrumentation}

LCMSMS analyses were performed using a Thermo Finnigan LCQ Deca XP Plus ion trap mass spectrometer with an electrospray ionization (ESI) source interfaced with a Surveyor autosampler and LC pump (Thermo Electron, San Jose, CA, USA).

\section{LCMSMS procedure}

Working calibrator and QC solutions were prepared by appropriate dilution of purchased methanolic drug stocks. Fifty microliters of calibrator working solution in $200 \mu \mathrm{L}$ blank urine produced final calibrator concentrations of $5,10,25,50,100,250,500$, and 1,000 ng/mL. Low-, medium-, and high-concentration QC samples were prepared at 15, 400, and $800 \mathrm{ng}$ / $\mathrm{mL}$ for BUP, $13.75,412.5$, and 825 for BUP-Gluc, and 75,400 , and $800 \mathrm{ng} / \mathrm{mL}$ for NBUP and NBUP-Gluc.

A $200 \mu \mathrm{L}$ urine aliquot, $50 \mu \mathrm{L}$ appropriate calibrator or control solution, $100 \mu \mathrm{L}$ internal standard working solution ( $400 \mathrm{ng} / \mathrm{mL} d_{3}$-NBUP and $200 \mathrm{ng} / \mathrm{mL} d_{4}$-BUP) and $1 \mathrm{~mL} 0.1 \%$ perchloric acid were combined. After having been vortexed for $10 \mathrm{~s}$, samples were applied to Strata-XC solid phase extraction (SPE) columns $(60 \mathrm{mg} / 3 \mathrm{~mL}$ ) (Phenomenex, Torrance, CA, USA) preconditioned with $1 \mathrm{~mL}$ methanol followed by $1 \mathrm{~mL} 0.1 \%$ perchloric acid. Cartridges were washed with $1 \mathrm{~mL} 2 \%$ formic acid and $1 \mathrm{~mL}$ methanol and dried under vacuum for 15 $\mathrm{min}$. Analytes were eluted with $5 \mathrm{~mL}$ methylene chloride-2-propanol-concentrated ammonium hydroxide (65:30:5) that was evaporated to dryness under nitrogen at $45^{\circ} \mathrm{C}$ with a Turbo Vap LV (Zymark, Hopkinton, MA, USA). Dried extracts were reconstituted in 100 $\mu \mathrm{L} 85: 15$ mobile phase $\mathrm{A}(0.1 \%$ formic acid and water) and mobile phase $\mathrm{B}(0.1 \%$ formic acid in acetonitrile) and $15 \mu \mathrm{L}$ was injected into the mass spectrometer.

Chromatographic separation was achieved with a Synergi Hydro-RP 80 A $(50 \mathrm{~mm} \times 2 \mathrm{~mm}, 4$ $\mu \mathrm{m}$ ) column with a $4 \mathrm{~mm} \times 2 \mathrm{~mm}$, identically packed, guard column (Phenomenex, Torrence, CA, USA) and gradient elution with 85:15 mobile phases A and B. The initial mixture, at a $200 \mu \mathrm{L} / \mathrm{min}$ flow rate, was decreased from 85 to $70 \%$ mobile phase A over $3 \mathrm{~min}$. The flow 
was increased to $300 \mu \mathrm{L} / \mathrm{min}$ and the amount of mobile phase A was decreased to $26 \%$ over 7 $\mathrm{min}$. The flow was then adjusted to $100 \mu \mathrm{L} / \mathrm{min}$, and the amount of mobile phase A was decreased to $15 \%$ over $10 \mathrm{~min}$ and held for $1 \mathrm{~min}$. The flow and mobile phase ratio were returned to the initial conditions over $1 \mathrm{~min}$, and the column was re-equilibrated for $8 \mathrm{~min}$, for a total run time of $20 \mathrm{~min}$.

Mass-spectral data were acquired in positive ion mode with the following ESI-MS parameters: sheath gas flow rate setting, 50; auxiliary gas flow rate setting, 10 ; spray voltage, $5.00 \mathrm{kV}$; and transfer capillary temperature, $250^{\circ} \mathrm{C}$. Precursor and product ion identification and $\mathrm{MS}^{n}$ optimization were established by directly infusing $1,000 \mathrm{ng} / \mathrm{mL}$ solutions of single analytes in methanol. Two scan events were performed for each analyte for quantification and identification purposes. The quantification scan event was selected ion monitoring of the precursor ion without fragmentation. For analyte identification, $\mathrm{MS}^{3}$ and $\mathrm{MS}^{2}$ fragmentation were monitored in full-scan mode. Each free analyte was identified by the presence of four characteristic ions from $\mathrm{MH}^{+}$fragmentation. Glucuronidated analytes were identified by $\mathrm{MS}^{3}$ fragmentation. First, $\mathrm{MH}^{+}$was fragmented cleaving the glucuronide moiety; the surviving molecule was further fragmented and two characteristic ions were monitored. Figure 1 displays full-scan mass spectra of each analyte, $\mathrm{MH}^{+}$, collision energies, and monitored ions.

\section{LCMSMS validation}

The method was validated for sensitivity, linearity, carryover, imprecision, recovery, extraction efficiency, matrix effect, process efficiency, matrix interference, dilution integrity, specificity, and stability. Sensitivity was assessed by establishing the limit of detection (LOD) and limit of quantification (LOQ) for each analyte. These parameters were evaluated with decreasing analyte concentrations in drug-fortified urine. The LOD was defined as the lowest concentration with acceptable chromatography, the presence of target and qualifier ions with signal-to-noise ratios of at least 3 , and a retention time within $\pm 0.2 \mathrm{~min}$ of the average calibrator retention time. The LOQ was the lowest concentration that met the LOD criteria with a signalto-noise ratio of at least 10 for all ions and acceptable accuracy and imprecision as defined below.

Linearity was determined by least-squares regression with a $1 / x$ weighting factor to compensate for heteroscedasticity. Acceptable linearity was achieved when the coefficient of determination was at least 0.98 and quantification of calibrators was within $\pm 20 \%$ of target at the LOQ and $\pm 15 \%$ at other concentrations. Lack of carryover was demonstrated by injecting internalstandard-fortified blank urine immediately after a sample spiked with $2,500 \mathrm{ng} / \mathrm{mL}$ of all analytes. Carryover was considered negligible up to $2,500 \mathrm{ng} / \mathrm{mL}$ if the calculated concentrations of analytes in the carryover QC sample were below the method LOD.

Imprecision, recovery, extraction efficiency, matrix effect, process efficiency, matrix interference, and stability were examined across the analytical range of the method with low-, medium-, and high-concentration QC samples. Target concentrations for low-, medium-, and high-concentration QC samples were 15, 400, and $800 \mathrm{ng} / \mathrm{mL}$ for BUP, 13.75, 412.5, and 825 for BUP-Gluc, and 75,400 , and $800 \mathrm{ng} / \mathrm{mL}$ for NBUP and NBUP-Gluc. Intra-assay imprecision was computed by quantifying five replicates of each QC concentration on each of six validation days and calculating the percent relative standard deviation (\%RSD) for that day; the highest \%RSD is reported. Interassay imprecision was calculated using one-way analysis of variance with Tukey's post hoc test to determine if there was a significant difference in the average concentrations calculated on each day. Intra-assay imprecision was required to be within $\pm 20 \%$. Recovery was acceptable if the mean calculated concentrations of 30 replicates tested over 6 days were within $\pm 20 \%$ of the nominal concentration. 
The extraction efficiency for each analyte was measured at each QC concentration. Blank urine was fortified with QC and internal standard solution before and after SPE. The percent extraction efficiency from urine was expressed as the mean analyte area of samples $(N=5)$ fortified with control solution before extraction divided by the mean area of samples $(N=5)$ with control solution added after SPE.

The matrix effect was assessed by comparing analyte peak areas in ten unique blank extracted urine samples fortified with QC and internal standard solutions after SPE with peak areas of samples at the same nominal concentrations prepared in an 85:15 mixture of mobile phase A and mobile phase B. Matrix suppression or enhancement, expressed as a percentage, was calculated as the ratio of average extracted urine peak area to average neat peak area.

The process efficiency examines the overall effect of the extraction efficiency and the matrix effect together on the quantification of analytes of interest. It was determined by comparing mean analyte peak areas of five samples fortified before SPE with the mean peak areas of five neat samples prepared in mobile phase at the same concentration.

Interference from endogenous matrix components was determined by spiking ten unique blank urine samples with BUP and metabolites at the method LOQ. Matrix interference was considered insignificant if imprecision and recovery at the LOQ were within $\pm 20 \%$.

Dilution integrity was evaluated by diluting urine samples $(N=3)$ containing $2,000 \mathrm{ng} / \mathrm{mL}$ of each analyte with blank urine to achieve a 1:4 dilution. Internal standard was added and samples were extracted as already described. Dilutional integrity was maintained if specimens were quantified within $\pm 20 \%$ of $500 \mathrm{ng} / \mathrm{mL}$.

Method specificity was demonstrated by adding high concentrations $(5,000 \mathrm{ng} / \mathrm{mL})$ of potentially interfering licit and illicit drugs to low-concentration QC samples. The following drugs and metabolites were examined: cocaine, benzoylecgonine, norcocaine, norbenzoylecgonine, ecgo-nine ethyl ester, ecgonine methyl ester, anhydroecgonine methyl ester, ecgonine, cocaethylene, norcocaethylene, $m$-hydroxycocaine, $p$-hydroxycocaine, $m$ hydroxybenzoylecgonine, $p$-hydroxybenzoylecgonine, methadone, 2-ethyl-5-methyl-3,3diphenyl-1-pyrroline, 2-ethyl-1,5-dimethyl-3, 3-diphenylpyrrolinium, methadol, $\Delta^{9}$ tetrahydrocannabinol, 11-hydroxy- $\Delta^{9}$-tetrahydrocannabinol, 11-nor-9-carboxy- $\Delta^{9}$ tetrahydrocannabinol, morphine, normorphine, morphine $3 \beta$-glucuronide, morphine $6 \beta$ glucuronide, codeine, norcodeine, 6-acetylmorphine, 6-acetylcodeine, hydrocodone, hydromorphone, oxycodone, noroxycodone, oxymorphone, norhydromorphone, noroxymorphone, $(R)-(+)$-cathinone, $( \pm)-N$-ethylamphetamine, 4-bromo-2,5dimethoxyphenethylamine, diazepam, lorazepam, oxazepam, alprazolam, imipramine, clomipramine, fluoxetine, norfluoxetine oxalate, paroxetine maleate, 7-aminoclonazepam, 7aminoflunitrazepam, 7-aminonitrazepam, clonidine, ibuprofen, pentazocine, caffeine, diphenhydramine, chlorpheniramine, brompheniramine, acetylsalicylic acid, acetaminophen, phencyclidine, bromazepam, clonazepam, flurazepam, nitrazepam, flunitrazepam, temazepam, nordiazepam, methamphetamine, amphetamine, 3,4methylenedioxymethamphetamine, 3,4-methylenedioxyethylamphetamine, 3,4methylenedioxyamphetamine, hydroxyamphetamine, hydroxymethamphetamine, 4hydroxy-3-methoxymethamphetamine, norephedrine, hydroxynorephedrine, nicotine, cotinine, hydroxycotinine, nornicotine, and norcotinine. In addition, urine specimens from six people known to have taken opiates and cocaine and from six people known to have smoked cannabis were fortified with low QC concentrations of BUP and metabolites and analyzed. The purpose of this testing was to ensure that naturally produced glucuronide and sulfate metabolites did not interfere in the method. Sufficient specificity was achieved if test samples were quantified within $\pm 20 \%$ of the low QC concentration. 
Analyte stability was investigated under a variety of conditions. Prepared specimen stability was determined by duplicate QC samples stored at $10^{\circ} \mathrm{C}$ on the autosampler 48 and $72 \mathrm{~h}$ after preparation. In addition, stability was tested for drug-fortified urine stored at room temperature $\left(22^{\circ} \mathrm{C}\right)$ for $16 \mathrm{~h}$, in the refrigerator $\left(4^{\circ} \mathrm{C}\right)$ for $72 \mathrm{~h}$, and after three freeze-thaw cycles.

\section{Proof of method}

Twenty-seven urine specimens from a pregnant woman treated with $18 \mathrm{mg}$ BUP/day were analyzed by the validated method. Specimens were collected as part of an Institutional Review Board approved clinical study on treatment of opioid dependence during gestation and participants provided written informed consent.

\section{Results}

The method was validated for sensitivity, linearity, carryover, imprecision, recovery, extraction efficiency, matrix effect, matrix interference, dilution integrity, specificity, and stability. Dynamic linear ranges and calibration results are shown in Table 1 . The LOD was $20 \mathrm{ng} / \mathrm{mL}$ for NBUP and NBUP-Gluc and $2 \mathrm{ng} / \mathrm{mL}$ for BUP and BUP-Gluc. The LOQ was $5 \mathrm{ng} / \mathrm{mL}$ for BUP and BUP-Gluc and $25 \mathrm{ng} / \mathrm{mL}$ for NBUP and NBUP-Gluc. A representative total ion chromatogram of blank urine is displayed in Fig. 2a. Selected ion monitoring chromatograms of precursor ions in urine fortified with BUP, NBUP, BUP-Gluc, and NBUP-Gluc at the LOQ are illustrated in Fig. 2b. No carryover was detected in a negative specimen injected immediately following analysis of a $2,500 \mathrm{ng} / \mathrm{mL}$ QC sample, indicating no carryover at this concentration.

Method validation results for imprecision and recovery are summarized in Table 2. Intra-assay imprecision was less than $16.6 \%$ for each of the four batches and interassay imprecision was less than $12.0 \%$ for all analytes at all concentrations. There was a statistically significant difference between daily mean concentrations for all analytes at different QC concentrations; however, the differences were less than $20 \%$ and were considered clinically insignificant. Recovery was $93.7-115.7 \%$ across the analytical range for all analytes.

Table 3 contains extraction efficiency, matrix effect, and process efficiency data. The extraction efficiency, evaluated at each QC concentration, ranged from 71.0 to $98.5 \%$. Average matrix suppression values of 2.2, 5.7, and 33.7\% were observed for BUP, BUP-Gluc, and NBUPGluc, respectively. NBUP demonstrated ion enhancement averaging $7.3 \%$ across concentrations. The process efficiency, which reflects the cumulative effects of the extraction efficiency and the matrix effect, was $57-122 \%$ with \%RSD no more than $26.3 \%$.

During method development an interference was noted in some blank urine specimens that potentially could affect quantification of NBUP and its conjugate; however, LOQ fortified blank urine samples were all quantified within $9.5 \%$ of target for all analytes, indicating that matrix interference did not affect quantification at the LOQ.

The ability of the method to accurately quantify specimens containing high concentrations of analytes was evaluated by diluting $2,000 \mathrm{ng} / \mathrm{mL}$ samples $(N=3)$ with blank urine to achieve a $1: 4$ dilution. All samples were quantified within $18.5 \%$ of the target concentration of $500 \mathrm{ng} /$ $\mathrm{mL}$, confirming dilution integrity.

Method specificity was demonstrated by adding high concentrations $(5,000 \mathrm{ng} / \mathrm{mL})$ of 69 potentially interfering licit and illicit drugs to low-concentration QC samples. Additionally, 12 authentic human urine specimens collected following cocaine, opiate, or cannabis use were fortified at low QC concentrations and analyzed. All test samples were quantified within $\pm 20 \%$ of target, indicating no interference with the four analytes of interest. 
Table 4 summarizes the stability of BUP and NBUP in urine, reported as the percentage difference between mean fresh control concentrations and mean concentrations following storage. Analytes were stable under all storage conditions with the percentage difference from fresh controls ranging from 83.7-119.9.

A chromatogram of a patient specimen containing all four analytes can be seen in Fig. 2c. All specimens $(N=27)$ contained NBUP, BUP-Gluc, and NBUP-Gluc, while only four had BUP concentrations above the LOQ. Mean concentrations were 7.0 $\pm 2.7,347.2 \pm 296.7,144.2 \pm 106.3$, and $1388.3 \pm 945.0 \mathrm{ng} / \mathrm{mL}$ for BUP, NBUP, BUP-Gluc, and NBUP-Gluc, respectively.

\section{Discussion}

Simultaneous quantification of nonpolar free and polar glucuronide-bound analytes can be complex. SPE requires a careful balance of polar and nonpolar solvent to achieve high extraction efficiency of free and bound drug while minimizing elution of polar matrix components. Separation by LC requires a column that can adequately retain glucuronide conjugates and provide sufficient resolution of analytes of interest. While this is not the first validated LCMSMS method for quantifying BUP, NBUP, and conjugated metabolites in urine, more extensive parameters for identifying analytes of interest and a more thorough validation are provided than previously published.

Table 5 outlines the validation performed on two previously published methods that simultaneously identified and quantified BUP, NBUP, BUP-Gluc, and NBUPGluc in urine as compared with the current research. All studies examined intraday and interday imprecision, recovery (accuracy), and stability in a similar manner. Previous methods reported lower LOQs than the current study (LOQs for BUP, BUP-Gluc, and NBUP-Gluc were $0.5 \mathrm{ng} / \mathrm{mL}$, and for NBUP were 1 and $2.5 \mathrm{ng} / \mathrm{mL}$ ) [20,21]; however, the current method determined the LOQs empirically, while the previous methods did not clearly describe how the LOQs were obtained. The upper LOQ was $50 \mathrm{ng} / \mathrm{mL}$ for Huang et al. [20] and $100 \mathrm{ng} / \mathrm{mL}$ for Liu et al. [21], much lower than the current upper LOQ of 1,000 ng/mL.

In addition to the more extensive method validation, the parameters for identifying analytes of interest are clearly stated and are more extensive in this study than in previously published studies. Previous methods quantified BUP and metabolites by monitoring the surviving protonated molecular ion $\left(\mathrm{MH}^{+}\right)[16,17]$ or by monitoring a selected transition [18-21], while operating in selected ion monitoring mode. BUP and metabolites are difficult to fragment $[20,22]$. In the current research, fragmentation was sometimes inconsistent. Accurate quantification was achieved by monitoring $\mathrm{MH}^{+}$in single $\mathrm{MS}$ selected ion mode instead of measuring transitions after fragmentation. Because fragmentation was inconsistent, ion ratios could not be used for identification purposes. Previous methods have used retention time alone [19], one or two transitions in multiple reaction monitoring mode [16,18], or three ions in fullscan mode [17] for identification purposes. Liu et al. [21] provided four tentative MSMS fragment ions for BUP and NBUP and two for BUP-Gluc and NBUP-Gluc, but did not describe how many ions were employed for identification. During method development, interference was noted in some blank urine specimens affecting quantification of NBUP and its conjugate at low concentrations; thus, the higher LOQ of $25 \mathrm{ng} / \mathrm{mL}$ for NBUP and NBUP-Gluc. Background interference was observed in two of six blank urine specimens investigated by Huang et al. [20], while other researchers reported no matrix interference using six to ten blank specimens [17-19].

The recovery and imprecision of the method was within $\pm 15 \%$ for most analytes and concentrations, except for the low QC concentration that met the $\pm 20 \%$ criterion. There were two exceptions: the NBUP maximum intraday imprecision was $15.4 \%$ at the high concentration 
and BUP recovery was $84.3 \%$ at the medium QC level. These values are similar to those reported by Favretto et al. [17], using the same ion trap instrument; BUP precision at the medium and high QC concentrations (1.00 and $10.00 \mathrm{ng} / \mathrm{mL}$, respectively) was greater than $15 \%$. During method development, all attempts were made to optimize assay performance, including imprecision and recovery. Once method validation began, actual data were reported as obtained for these parameters. Acceptance criteria for imprecision and recovery for LCMSMS are changing with developments in the field; \pm 20 is the acceptance criterion for $\%$ RSD and recovery for our clinical assays. In addition, calibrators must be calculated against the curve response factor with concentrations within $\pm 20 \%$ of target. In fact, all met $\pm 15 \%$ of target, except for the LOQ that was within $\pm 20 \%$.

A variety of techniques can evaluate extraction efficiency, matrix effect, and process efficiency. The current study employed three sample types to evaluate these parameters. The first sample was urine spiked with analyte and internal standard prior to SPE (tube 1). The second sample was blank urine that was processed by SPE prior to the addition of drug and internal standard (tube 2). Finally, neat samples consisting of analyte and internal standard in the mobile phase were prepared (tube 3 ). The ratio of tube 1 to tube 2 represents the extraction efficiency. The tube 2 to tube 3 ratio determines the matrix effect, while the ratio of tube 1 to tube 3 expresses the overall process efficiency. Previous methods did not report all three of these parameters.

Liu et al. [21] compared directly injected drug-free urine samples and water, with postcolumn infusion of a mixture of analytes of interest to identify the matrix effect. There was a 35\% decrease in the signal with urine compared with water, indicating ion suppression. Next, the experiment was repeated with urine samples that had undergone SPE. The signal was suppressed by 7\%, indicating that SPE effectively removed most interfering matrix components. Huang et al. [20] did not report a matrix effect in their method. Hegstad et al. [19] also employed $d_{3}$-NBUP as an internal standard for BUP-Gluc and NBUP-Gluc in a method evaluating only the glucuronidated compounds in human urine. Variation in the matrix effect of six different urine pools was reported as \%RSD and was calculated for the mean peak height ratio between the analyte of interest and the internal standard. Determined by this method, \%RSD ranged from 22.5 to $28.1 \%$ for BUP-Gluc and NBUP-Gluc. Matrix effects were $234-253 \%$ for BUP-Gluc, $85-87 \%$ for NBUP-Gluc, and $144-152 \%$ for $d_{3}$-NBUP.

In the current method, NBUP-Gluc was poorly retained by the column and was eluted with polar matrix components increasing the matrix effect. While structurally similar deuterated internal standards compensated for the matrix effect (BUP and NBUP), deuterated glucuronide internal standards were not available. Matrix suppression of up to $38.2 \%$ was observed for NBUP-Gluc. To evaluate the variance in the matrix effect in ten different urine samples, the NBUP-Gluc area for samples fortified after SPE was divided by the mean area for ten neat samples at the same concentration and \%RSD was calculated. \%RSD was 20.3-26.3\%. When calculations were performed according to the method of Hegstad et al. [19] that incorporated the deuterated internal standard area, \%RSD was 17.1, 14.1, and 16.0\% at the low, medium, and high concentrations, respectively, indicating that the internal standard helped compensate for the observed matrix effect. \%RSD was less than $15 \%$ for ratios of the other analytes to the internal standard at all concentrations. Additionally, matrix interference was evaluated with the same blank urine samples at the LOQ, and all were quantified within $\pm 20 \%$, indicating that variable matrix effect did not affect quantification.

Liu et al. [21] reported "extraction recovery" of 93.6-102.2\% for analyte peak area ratios between fortified urine and water samples. This is not a measure of SPE efficiency since it does not compare samples fortified before and after SPE. Huang et al. [20] reported mean extraction efficiencies of 69.6, 84.3, 111, and 102\% for BUP, NBUP, BUP-Gluc, and NBUPGluc, respectively, with a technique similar to the one currently defined as process efficiency, 
although it is unclear if these results were for urine or plasma. Separate evaluations of extraction efficiency and the matrix effect were not performed. The process efficiency in the current method was 91.9, 82.6, 104.8, and 58.6\% for BUP, NBUP, BUP-Gluc, and NBUP-Gluc, respectively.

The distribution of BUP and metabolites in authentic patient specimens was in agreement with previously published reports that reported the majority of BUP excreted in urine is in the form of NBUP-Gluc [19-21]. The extent of free BUP excretion in urine varies in different publications. Cone et al. [23] reported no free BUP with a method LOQ of $10 \mathrm{ng} / \mathrm{mL}$, while Liu et al. [21] reported much higher concentrations of 8.2-30.5 ng/mL (method LOQ of 0.5 $\mathrm{ng} / \mathrm{mL}$ ). Attempts to improve the LOQ of the current method by increasing the sample volume were unsuccessful because of increased endogenous matrix components, negating any improvement from increased urine amount. Also, a higher specimen volume would have greatly increased the number of specimens with NBUP-Gluc concentrations exceeding the upper $L O Q$ requiring dilution.

There are several limitations of the current method. The higher LOQ for NBUP and NBUPGluc could result in a lower frequency of these analytes in urine; however, in the individual's urine specimens $(N=27)$ collected over 9 weeks and tested by the method, $100 \%$ tested positive for NBUP and NBUP-Gluc, indicating that the method is sufficient for quantifying these analytes in BUP-maintained patients. Also, no deuterated internal standards were available for the glucuronide conjugates, making it difficult to compensate for matrix suppression that could lead to underestimation of urine concentrations.

\section{Conclusions}

The current method improves on previously published LCMSMS methods for the quantification of BUP, NBUP, BUP-Gluc, and NBUP-Gluc in urine by including a thorough method validation and clearly describing identification criteria and procedures to quantify analytes of interest. Additionally, carryover, dilution integrity, and specificity with respect to potentially interfering exogenous analytes were included in the current validation but not in previously published reports. Ensuring lack of carryover confirms that a high-concentration specimen will not contribute to the quantification of the next injection. Proving that dilution does not affect quantification is essential for obtaining accurate results for high-concentration specimens, and specificity tests prove that other commonly encountered licit and illicit drugs will not affect the integrity of the results. This method will be employed to analyze over 500 urine specimens collected in a clinical study investigating BUP as a substitution therapy for opioid treatment during pregnancy.

\section{References}

1. Jenkinson RA, Clark NC, Fry CL, Dobbin M. Addiction 2005;100:197-205. [PubMed: 15679749]

2. Obadia Y, Perrin V, Feroni I, Vlahov D, Moatti JP. Addiction 2001;96:267-272. [PubMed: 11182872]

3. Aalto M, Halme J, Visapaa JP, Salaspuro M. Subst Use Misuse 2007;42:1027-1028. [PubMed: 17613961]

4. Bottcher M, Beck O. J Anal Toxicol 2005;29:769-776. [PubMed: 16356333]

5. De Giovanni N, Fucci N, Scarlata S, Donzelli G. Clin Chem Lab Med 2005;43:1377-1379. [PubMed: 16309376]

6. Cirimele V, Etienne S, Villain M, Ludes B, Kintz P. Forensic Sci Int 2004;143:153-156. [PubMed: 15240036]

7. Cirimele V, Kintz P, Lohner S, Ludes B. J Anal Toxicol 2003;27:103-105. [PubMed: 12670004]

8. Debrabandere L, Van Boven M, Daenens P. J Forensic Sci 1995;40:250-253. [PubMed: 7602287] 
9. Hand CW, Ryan KE, Dutt SK, Moore RA, O'Connor J, Talbot D, McQuay HJ. J Anal Toxicol 1989;13:100-104. [PubMed: 2733386]

10. Blom Y, Bondesson U. J Chromatogr 1985;338:89-98. [PubMed: 4019659]

11. George S, George C, Chauhan M. Forensic Sci Int 2004;143:121-125. [PubMed: 15240031]

12. Lange WR, Fudala PJ, Dax EM, Johnson RE. Drug Alcohol Depend 1990;26:19-28. [PubMed: 2209411]

13. Lisi AM, Kazlauskas R, Trout GJ. J Chromatogr B Biomed Sci Appl 1997;692:67-77. [PubMed: 9187385]

14. Cone EJ, Gorodetzky CW, Yousefnejad D, Darwin WD. J Chromatogr 1985;337:291-300. [PubMed: 3838755]

15. Vincent F, Bessard J, Vacheron J, Mallaret M, Bessard G. J Anal Toxicol 1999;23:270-279. [PubMed: 10445490]

16. Kronstrand R, Selden TG, Josefsson M. J Anal Toxicol 2003;27:464-470. [PubMed: 14607001]

17. Favretto D, Frison G, Vogliardi S, Ferrara SD. Rapid Commun Mass Spectrom 2006;20:1257-1265. [PubMed: 16550495]

18. Fox EJ, Tetlow VA, Allen KR. J Anal Toxicol 2006;30:238-244. [PubMed: 16803661]

19. Hegstad S, Khiabani HZ, Oiestad EL, Berg T, Christophersen AS. J Anal Toxicol 2007;31:214-219. [PubMed: 17555645]

20. Huang W, Moody DE, McCance-Katz EF. Ther Drug Monit 2006;28:245-251. [PubMed: 16628138]

21. Liu AC, Lin TY, Su LW, Fuh MR. Talanta 2008;75:198-204. [PubMed: 18371868]

22. Moody DE, Laycock JD, Spanbauer AC, Crouch DJ, Foltz RL, Josephs JL, Amass L, Bickel WK. J Anal Toxicol 1997;21:406-411. [PubMed: 9323518]

23. Cone EJ, Gorodetzky CW, Yousefnejad D, Buchwald WF, Johnson RE. Drug Metab Dispos 1984;12:577-581. [PubMed: 6149907] 
a Buprenorphine Fragmentation: $468.4 @ 36 \%^{*} \rightarrow 396,414,426,450$

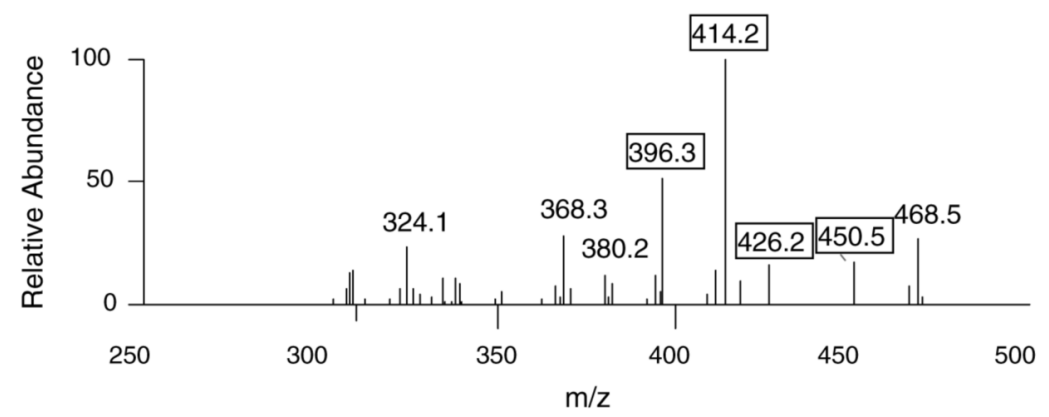

b Norbuprenorphine Fragmentation: $414.3 @ 33 \%{ }^{*} \rightarrow 340,364,382,396$

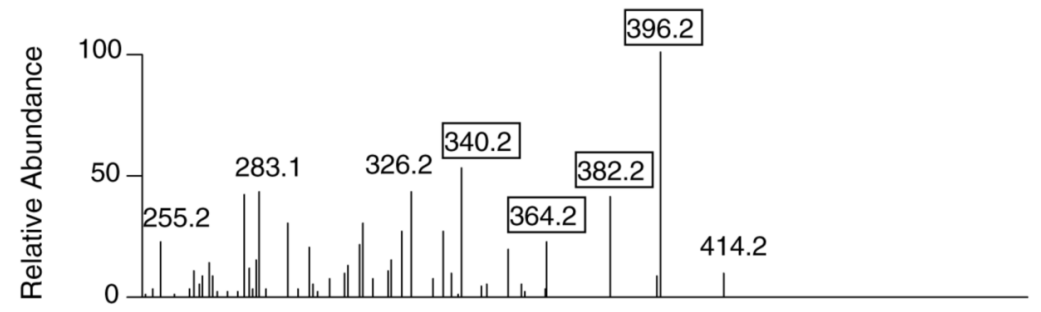

C Buprenorphine Glucuronide Fragmentation: 644.0 @ 29\%* $\rightarrow 468.0 @ 36 \% \%^{*} \rightarrow 414,396$

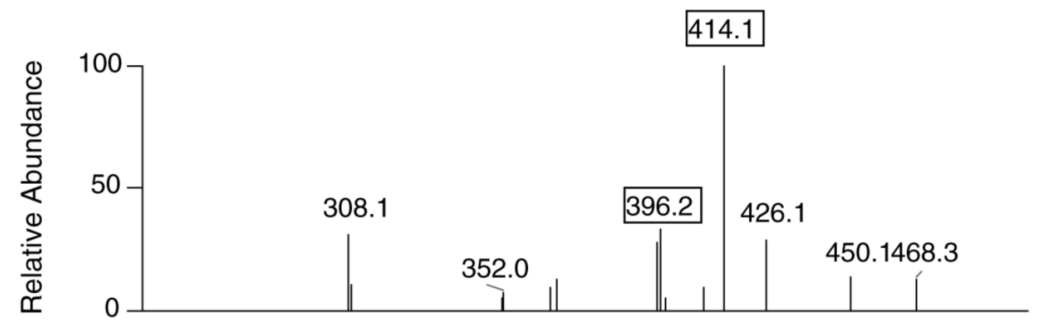

d Norbuprenorphine Glucuronide Fragmentation: $590.0 @ 29 \%{ }^{*} \rightarrow 414.2 @ 32 \%{ }^{*} \rightarrow 396,340$

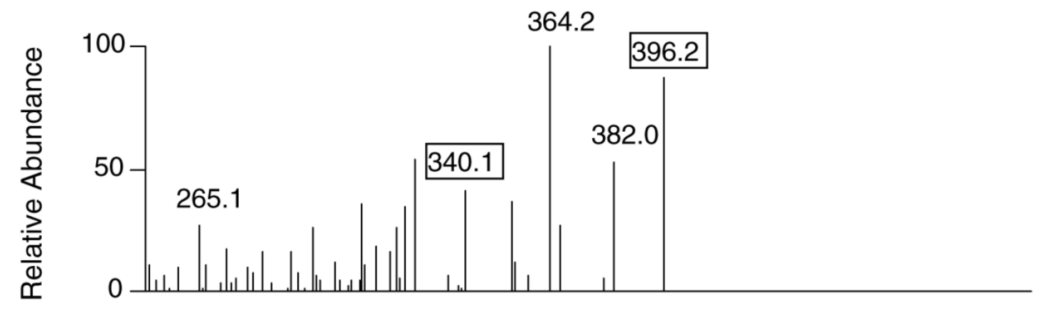

*Normalized collision energy

Fig. 1.

Full-scan $\mathrm{MS}^{2}$ mass spectra of buprenorphine (BUP) and norbuprenorphine (NBUP) and $\mathrm{MS}^{3}$ mass spectra of buprenorphine glucuronide (BUP-Gluc) and norbuprenorphine glucuronide (NBUP-Gluc) 

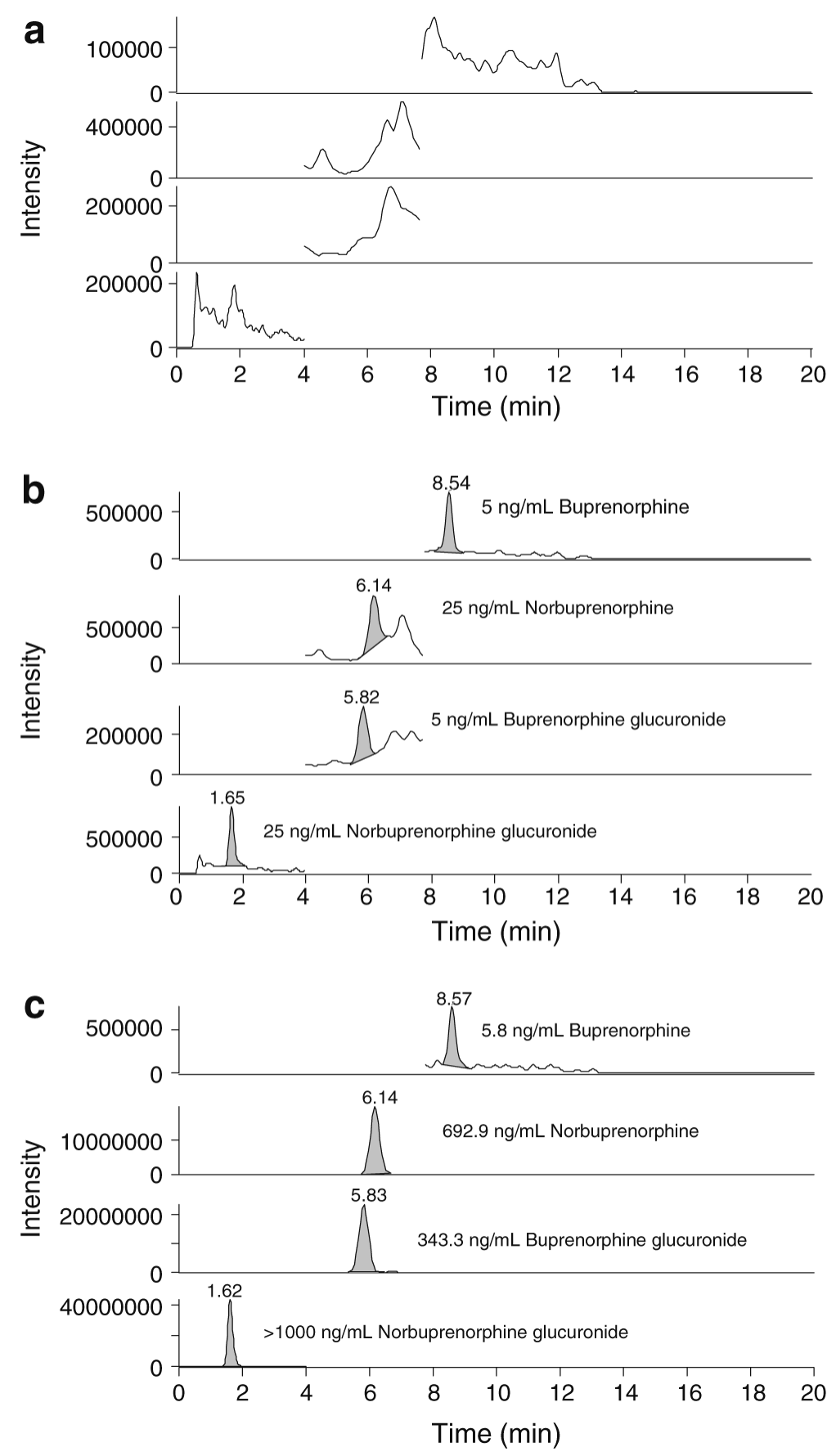

Fig. 2.

Selected ion monitoring chromatograms of precursor ions of $\mathbf{a}$ extracted blank urine, $\mathbf{b}$ urine fortified with $5 \mathrm{ng} / \mathrm{mL}$ BUP and BUP-Gluc and $25 \mathrm{ng} / \mathrm{mL}$ NBUP and NBUP-Gluc, and $\mathbf{c}$ representative urine specimen containing 5.8, 692.9, 343.3, and more than $1000 \mathrm{ng} / \mathrm{mL}$ BUP, NBUP, BUP-Gluc, and NBUP-Gluc, respectively. NBUP-Gluc was diluted 1:4 with blank urine and reanalyzed to achieve a final concentration of $1,356.4 \mathrm{ng} / \mathrm{mL}$ 
Table 1

Liquid chromatography-tandem mass spectrometry (LCMSMS) calibration data for buprenorphine, norbuprenorphine, and conjugated metabolites in urine ( $N=7$ curves)

\begin{tabular}{lllll}
\hline Compound & Linear range (ng/mL) & Mean slope ${ }^{\boldsymbol{a}}$ & Mean intercept $^{\boldsymbol{a}}$ & $\begin{array}{l}\text { Coefficient of } \\
\text { determination, } \\
\boldsymbol{R}^{2}\end{array}$ \\
\hline Buprenorphine & $5-1,000$ & & & $0.991-0.998$ \\
Buprenorphine glucuronide & $5-1,000$ & $0.0107(0.0003)$ & $0.0066(0.0054)$ & $0.987-0.999$ \\
Norbuprenorphine & $25-1,000$ & $0.0093(0.0009)$ & $0.0036(0.0061)$ & $0.989-0.999$ \\
Norbuprenorphine glucuronide & $25-1,000$ & $0.004(0.0002)$ & $0.079(0.0275)$ & $0.987-0.997$ \\
\hline
\end{tabular}

$a_{\text {The standard error is given in parentheses. }}$ 
Kacinko et al.

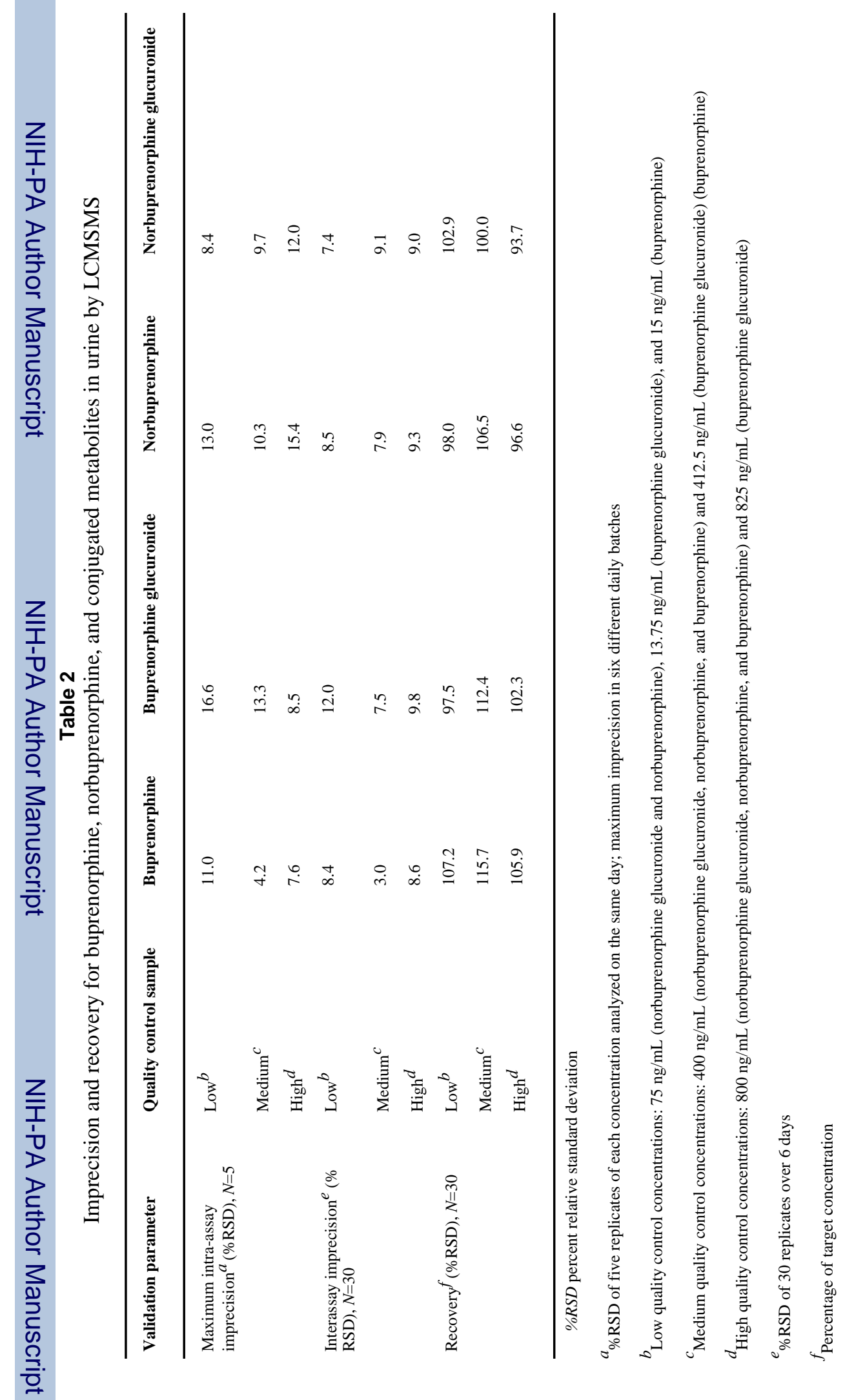


Kacinko et al.

Page 15

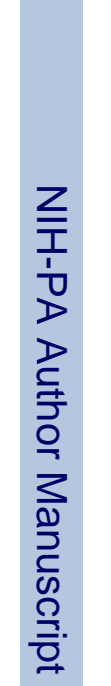

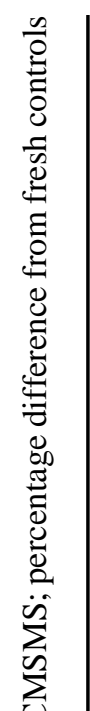

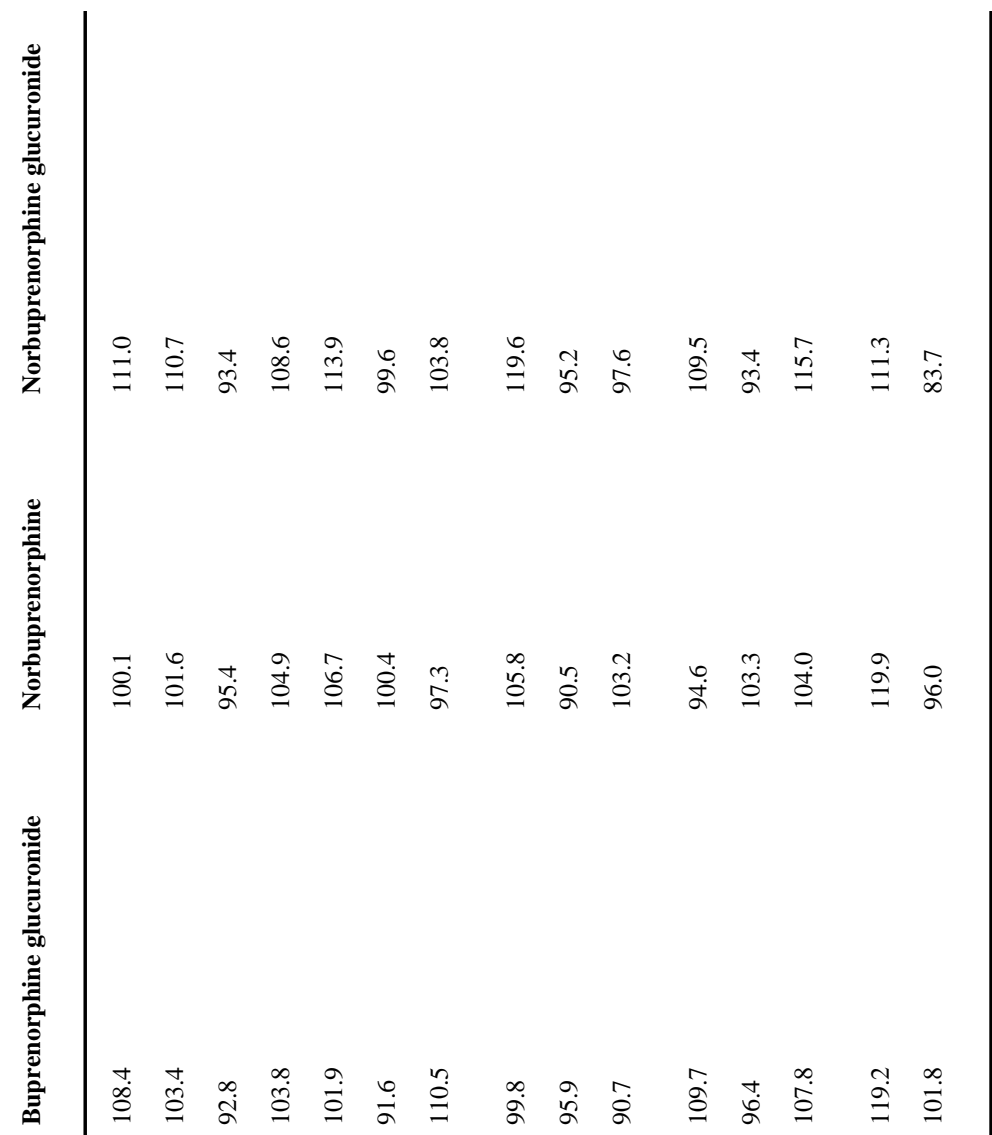

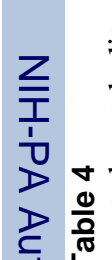

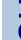

.

돌.

.

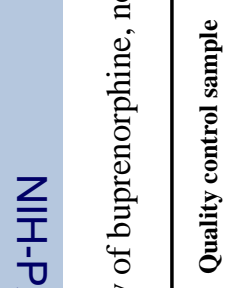

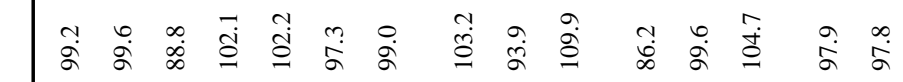

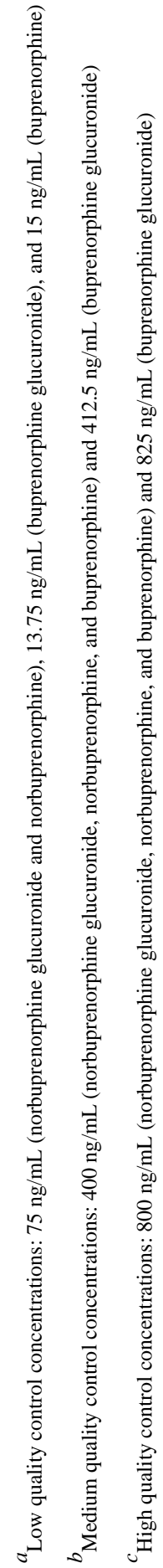
Anal Bioanal Chem. Author manuscript; available in PMC 2009 November 1. 
Table 5

Comparison of evaluated method validation parameters between the current study and two previously published reports

\begin{tabular}{lccc}
\hline & Current study & Liu et al. [21] $^{\boldsymbol{a}}$ & Huang et al. [20] $^{\boldsymbol{b}}$ \\
\hline Sensitivity & $\mathrm{X}$ & $\mathrm{X}$ & $\mathrm{X}$ \\
Linearity & $\mathrm{X}$ & $\mathrm{X}$ & $\mathrm{X}$ \\
Carryover & $\mathrm{X}$ & $\mathrm{X}$ & $\mathrm{X}$ \\
Imprecision & $\mathrm{X}$ & $\mathrm{X}$ & $\mathrm{X}$ \\
Recovery & $\mathrm{X}$ & $\mathrm{X}$ & $\mathrm{X}$ \\
Extraction efficiency & $\mathrm{X}$ & $\mathrm{X}$ & $\mathrm{X}$ \\
Matrix effect & $\mathrm{X}$ & & $\mathrm{X}$ \\
Process efficiency & $\mathrm{X}$ & $\mathrm{X}$ & \\
Matrix interference & $\mathrm{X}$ & $\mathrm{X}$ \\
dilution & $\mathrm{X}$ & $\mathrm{X}$ & \\
Specificity & $\mathrm{X}$ & \\
Stability & $\mathrm{X}$ & & \\
\hline
\end{tabular}

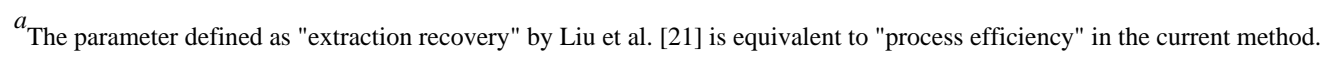

${ }^{b}$ The parameter defined as "recovery" by Huang et al. [20] is equivalent to "process efficiency" in the current study and was determined for plasma, not urine. 